\title{
La Reforma de las Administraciones Públicas en España tras la Constitución de 1978
}

Julio Seage *

\section{Problema planteado}

Los Recursos humanos de la Administración Pública de España estaban compuestos en 1994 por 2.256 .063 personas, esti- madas las tres Administraciones y las empresas públicas (Cuadro 3). Esta inmensa maquinaria tenía un coste del orden de 6,9 billones de ptas. (un 23,1\% del Presupuesto). Si nos ceñimos a la Administración Central, el aparato estatal englobaba 873.702 personas y costaba del orden de 3,4 billones de ptas. (Cuadros $1,2$ y 3$)$.

\section{Cuadro 1}

\section{Presupuesto de las Administraciones Públicas en 1993}

I. AA.PP. CENTRALES DEI ESTADO

\begin{tabular}{cccc}
$\begin{array}{c}\text { Presupuesto } \\
\text { Todos los cap. } \\
\text { (Millones Ptas.) }\end{array}$ & $\begin{array}{c}\text { Capitulo I } \\
\text { Personal } \\
\text { (Millones Ptas.) }\end{array}$ & \% Cap. I/Pto. & \% T. Cap. I \\
\hline 24.613 .516 & 3.445 .496 & 14,0 & 50,0
\end{tabular}

II. COMUNIDADES AUTONOMAS

\begin{tabular}{cccc}
$\begin{array}{c}\text { Presupuesto } \\
\text { Todos los cap. } \\
\text { (Millones Ptas.) }\end{array}$ & $\begin{array}{c}\text { Capitulo I } \\
\text { Personal } \\
\text { (Millones Ptas.) }\end{array}$ & \% Cap. I/Pto. & \% T. Cap. I \\
\hline 6.944 .607 & 2.289 .768 & 33,0 & 33,3
\end{tabular}

III. CORPORACIONES LOCALES

\begin{tabular}{cccc|}
$\begin{array}{c}\text { Presupuesto } \\
\text { Todos los cap. } \\
\text { (Millones Ptas.) }\end{array}$ & $\begin{array}{c}\text { Capitulo I } \\
\text { Personal } \\
\text { (Millones Ptas.) }\end{array}$ & \% Cap. 1/Pto. & \% T. Cap. J \\
\hline 4.363 .453 & 1.149 .771 & 26,4 & 16,7 \\
35.921 .576 & 6.885 .035 & 19,2 & 100,0 \\
29.826 .061 & 6.885 .035 & 23,1 & \\
\hline
\end{tabular}

Fuente: Informe Económico-Financiero de las Administraciones Territonales en 1993, pág. 316. 


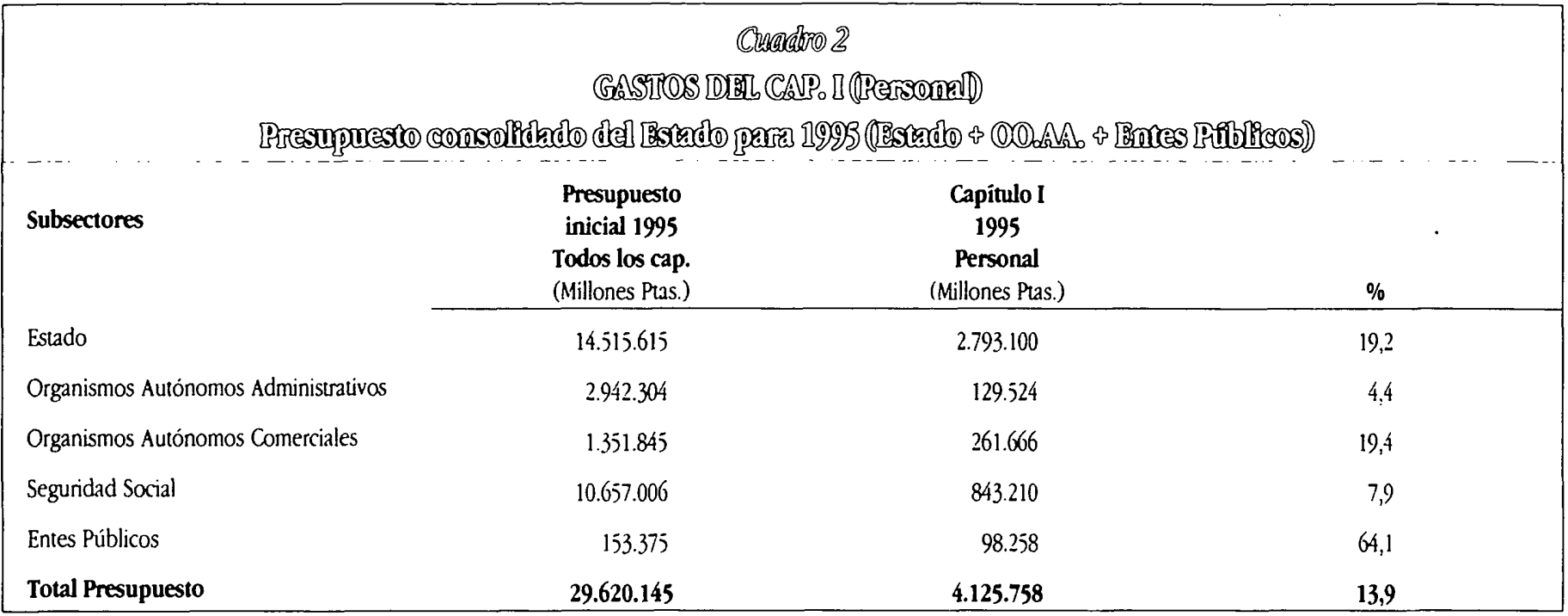

(Datos del libro Presentación del Proyecto de Presupuestos Generales del Estado 1995).

\begin{tabular}{|c|c|c|c|c|c|}
\hline \multicolumn{6}{|c|}{ 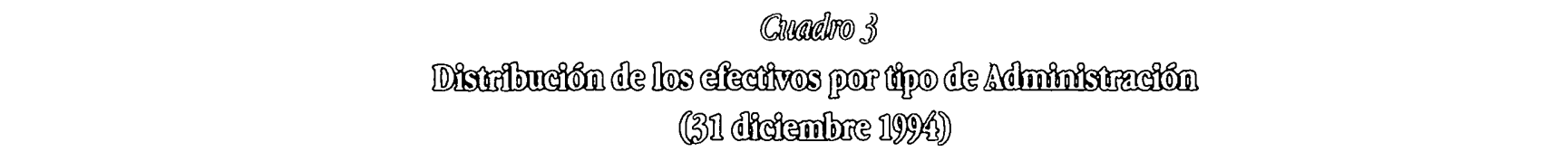 } \\
\hline & $\begin{array}{l}\text { Admón. } \\
\text { Central }\end{array}$ & CC.AA. & $\begin{array}{l}\text { Admón. } \\
\text { Local }\end{array}$ & Otros & Total \\
\hline Ministerios y OO.AA. (1) & 314.451 & & & & 314.451 \\
\hline Administración de Justicia & 38.25 毛 & & & & 38.254 \\
\hline Fuerzas Armadas & 73.327 & & & & 73.327 \\
\hline Fuerzas de Seguridad del Estado & 117.958 & & & & 117.958 \\
\hline Efectivos Adminisıración Local & & & 371.456 & & 371.456 \\
\hline Instituciones Sanitarias & 130.537 & 176.033 & & & 306.570 \\
\hline Agencia Estatal Admón. Tributaria & 27.124 & & & & 27.124 \\
\hline Entes Públicos y Socied. Estatales & & & & 372.643 & 372.643 \\
\hline Universidades (2) & 38.503 & 38.492 & & & 76.995 \\
\hline CC.AA. Personal Admón. y otros (3) & & 208.225 & & & 208.225 \\
\hline Efect. de Enseñanza no Universitaria & 133.548 & 217.069 & & & 350.617 \\
\hline Total & 873.702 & 639.819 & 371.456 & 372.643 & 2.257 .620 \\
\hline
\end{tabular}

(1) Comprenden 192.251 efectivos de funcionarios y 122.200 efectivos de personal laboral.

(2) Comprende Personal docente y no docente.

(3) Comprende: Funcionarios de carrera Interinos, Eventuales, Sanitarios Locales, Contratados Administratıvos y Personal laboral.

A lo largo de los últimos años, esta enorme maquinaria ha sido objeto de fuertes críticas por su incapacidad para resolver adecuadamente los problemas de los ciudadanos y por su escasa eficiencia.
Pero no sólo en España, también en la mayoría de los países occidentales se venían poniendo de relieve los problemas derivados de la inadecuación de las Administraciones Públicas. Esta inadecuación se manifestaba en que el modelo burocrático tradicional, nacido para dirigir una administración de potestades, no se adaptaba a la actual administración prestadora de 
servicios. Frente al procedimiento jurídico reglado o la jerarquización, se acentuaban aspectos tales como la consecución de resultados, la responsabilidad de los gestores o la calidad del servicio prestado.

\section{Los programas de "modernización" (1983-1996)}

\subsection{Experiencias de otros paises de la OCDE}

Quizás, la característica más relevante de la política del gasto público de personal de la Administración espanola de los años 80 ha sido su rapidísimo crecimiento en contraste con lo que sucedía en otros países de la OCDE, que en ese decenio iniciaron medidas de reducción de las plantillas de personal. Mientras la mayoría de los países de la OCDE redujeron, a partir de 1985, el empleo en sus Administraciones en relación con el empleo total, en España creció del 11,3\% (1980) al 15,2\% (1990).

Justamente, estos países de la OCDE tomaron medidas de reforma en la citada década para hacer más eficiente el sector público. Estas medidas abarcan un amplio abanico, desde las privatizaciones de empresas a las reducciones de personal, desde medidas de eficiencia a la profesionalización de directivos y desde revisiones en el binomio planificación-presupuesto a la flexibilización de los sistemas de función pública.

\section{Rasgos comunes}

En este proceso de reformas destacan las abordadas por el Reino Unido, Canadá o Francia. A pesar de sus diferencias, tienen una serie de rasgos comunes, entre los que destacan los siguientes (Instituto de Estudios Fiscales, 1993):

Papel del sector público: En general se realizaron revisiones de su cometido, que culminaron, en algunos casos, con amplios programas de privatización, sobre todo en el ámbito de las empresas públicas.

Función Pública: La modemización de las Administraciones Públicas ha pasado, en todos los casos, por una revisión de la Función Pública, reforzando su profesionalización, variando sus estructuras para adaptarlas a los cambios tecnológi$\cos$ y a los nuevos diseños organizativos, y reajustando las plantillas, a través de planes de reubicación de personal, formación, reciclaje, jubilaciones voluntarias, etc.
Organización: Todas las reformas apuestan por una mayor descentralización de los servicios, potenciando unidades de responsabilidad. Su existencia no implica, sin embargo, que hayan de ser objeto de un tratamiento especial en aspectos de procedimientos o de selección de personal, con carácter general. Su existencia favorece que en las actividades del Estado que van más allá de la simple regulación sea más fácil la aplicación de las técnicas de gestión por objetivos y que se mejore la eficiencia en la gestión.

Evaluación de politicas priblicas: Se muestra una preocupación especial por la gestión de los recursos públicos, en el sentido de que éstos se destinen a satisfacer necesidades debidamente evaluadas; se elijan las alternativas más favorables desde el punto de vista de sus beneficios y costes, y que en la selección de las opciones de gasto se introduzcan criterios de prioridad. Todo ello acompañado de una mayor eficacia en la gestión.

Fruto de esta preocupación es el reforzamiento de la evaluación de políticas. Ésta extiende a los sectores sociales la aplicación de técnicas racionales de análisis reservadas originariamente a sectores económicos. De igual modo, va más allá de la planificación tradicional, favoreciendo que el acercamiento entre los destinatarios de las políticas y los gestores de las mismas sea lo más elevada posible, evitando las políticas no consensuadas.

Programación plurianual y presupuesto: Se refuerza la programación plurianual y los acuerdos, contratos y convenios con las unidades de responsabilidad. De este modo, el Presupuesto es el último ejercicio de un proceso en el que se han definido la entidad de las políticas, sus prioridades y los cursos de acción posibles para un período plurianual. Sólo resta valorar cuánto cuestan las actuaciones anuales que exige el cumplimiento de los objetivos.

Potenciación de la función directiva: Los directivos son el recurso más valioso de las empresas. La incorporación de la función gerencial es absolutamente necesaria, si bien será necesario cambiar el perfil del funcionario directivo. La función gerencial pública exige un directivo que desarrolle políticas públicas y que tenga autoridad para gestionar recursos en función de unos resultados. Al lado del directivo político aparece el directivo profesional con campos de actuaciones diferentes, aunque coordinados. Al primero se le encomienda la función política y al segundo la función gerencial operativa y presupuestaria. A su vez, la selección de ambos es diferente, política el primero y profesional el segundo.

Además se ha ampliado el campo de reclutamiento de los directivos. En Italia esta ampliación se extendió a personas originarias de los sectores de investigación, de enseñanza superior 
y de organismos públicos y privados. En Gran Bretaña y Holanda el acceso a puestos directivos también se ha extendido a profesionales de empresas privadas, además del Civil Service (Cruz Ferrer, 1984; Guy Peters, 1994; Hood Cr., 1994; SMTth, BRUCE, 1984).

\section{Las reformas en Gran Bretaña}

El Gobierno conservador británico fijó como gran objetivo la reducción de gasto público. Para ello trató de reducir el tamaño del Sector Público y de mejorar la eficiencia de los servicios públicos. Las medidas concretas que puso en marcha fueron, además de las privatizaciones, la Financial Management Iniciative, Next Steps y las Cartas del Ciudadano.

La Unidad de Eficiencia trataba de identificar los objetivos de cada unidad, estimular a los Departamentos a realizar análisis de eficiencia y a difundir los resultados. Realizó sus análisis en algunos Departamentos que sirvieron para demostrar cómo estudios realizados en un corto espacio de tiempo podían dar lugar a mejoras de eficiencia. Esta Unidad impulsó la creación de FMI y del documento Next Steps.

La F.M. Iniciative nace en 1982 para promover en los Departamentos un cambio en la forma de dirigir. Se pedía a los directivos:

- Una definición de objetivos y de medios para evaluar resultados.

- Una responsabilidad sobre los resultados.

Para apoyar al F.M.I. y a los Departamentos se crea la Unidad de dirección financiera (F.M.U.), compuesta por 12 personas provenientes de la Administración y de la empresa privada. Esta unidad se centró en tres aspectos:

- Desarrollo de sistemas de información para la alta dirección.

- Utilización del presupuesto como instrumento de control y de mejora de la actividad.

- La gestión del sistema de gasto mediante el examen de los Departamentos y de los organismos que dependen de ellos.

Los resultados de esta primera fase, en 1988 , se resumen en una reducción del tamaño de la Administración en un 19\%, un ahorro de un billón de libras y en la mejora de los sistemas de información y presupuestación.

En este punto, la Unidad de Eficiencia señaló como problemas básicos de la Administración británica el centralismo y la burocratización. Para ello se propuso como solución la separación entre las actividades reguladoras y las ejecutivas, mediante la creación de las Agencias Ejecutivas. En 1987, la Unidad de Eficiencia presentó a la Primera Ministra el documento Next Steps que propuso creación de Agencias para lograr una mejora de la eficiencia de la Administración Pública. Para desarrollar este proyecto se creó un pequeño equipo de personas dirigido por un Director de Proyectos. A su vez se crearon dos grupos de apoyo: uno, compuesto por los Ministerios horizontales y otro, del que forman parte los representantes de los Departamentos.

Los criterios para decidir la creación de una Agencia son cuatro:

a) Análisis de las funciones de la futura Agencia y decisión sobre su importancia.

b) Estudiar si la actividad periódica es realizada en el sector privado o si podría contratarse con una firma privada.

c) Evaluar si las funciones de tipo ejecutivo son separables de las de carácter políico.

d) La independencia financiera respecto del Ministerio vía precios o vía límite financiero, dentro del cual podría operar la Agencia, (Butter, 1993; InstTuto de Estudios FISCALES, 1993; MÉNY, 1994).

Desde el punto de vista de la organización, la mayoría de las Agencias son financiadas por el Presupuesto del Ministerio del que dependen. Los ingresos provenientes de las tasas son ingresados en un fondo central y los gastos son sometidos a límites. Sin embargo, un pequeño número de agencias que pueden cubrir sus gastos con sus ingresos, pueden ser dispensadas del límite de gastos. En este caso intervienen otros métodos de eficacia y de control de gestión. El personal de las agencias que no requiere diploma universitario puede ser seleccionado directamente por las Agencias. El resto del personal debe ser funcionario del Civil Service. Los acuerdos-marco dan a las Agencias una cierta libertad para la definición de categorías profesionales, pero en cambio no pueden salirse de los escalones salariales del Civil Service, (STEVENS, A., 1990).

Un tercer bloque de reformas lo constituyen las Cartas del ciudadano. Éstas constituyen un conjunto de iniciativas de reforma a largo plazo -destinadas a ajustar cuando sea posible los servicios públicos a los deseos de los ciudadanos y a mejorar su calidad global. (INSTTUUTO DE Estudios FisCALES, 1993). Las cartas abordan problemas de calidad, elección y regulación de los servicios públicos. Adoptan para ello medidas para solucionar los problemas. Entre éstas destacan la información acerca de los servicios públicos y los requisitos mínimos de calidad, la introducción de unidades de inspección de calidad y la utilización de análisis de mercado para incentivar a la 
dirección de los centros, señalando cuáles prestan un servicio más eficiente.

\section{Las reformas en Suecia}

En Suecia comenzó un profundo proceso de ajuste en el sector público que se consideró necesario por varias razones: desempleo, que alcanzaba el $12 \%$ de la población activa, enorme déficit presupuestario equivalente al $12 \%$ del PIB y deuda pública del $90 \%$ del PIB. Una última razón apunta a la globalización de la economía a largo plazo. El aumento de la competitividad entre naciones que siguió a la desregulación del sistema económico en los años 80 y a la libertad de movimiento de capitales en el ámbito internacional, modificaron el contexto del sector público sueco. Como señala Lennart Gustafsson *con una economía universalizada, un país no puede soportar la carga que supone un sector público ineficaz y sobredimensionado. Tal país perdería inevitablemente competitividad respecto al resto. (GusTAFSSON, 1995).

Como consecuencia de esta situación, la cuestión se centra en Suecia no sólo en el nivel de los servicios que presta la Administración y de la eficacia con que los presta, sino también en la forma misma del Estado de Bienestar. A diferencia de la polémica sobre la modernización de la Administración de hace diez años, ahora se discute sobre aspectos estructurales de fondo. Esto quiere decir que la proporción del PNB destinada a gasto público debe disminuir y que el Estado debe dejar más funciones al mercado.

De acuerdo con estos criterios se proponen una serie de medidas para la reforma de la Administración.

\section{a) Cambios estructurales}

En los años 80 se iniciaron controles del nivel central, realizados por comités nombrados por el Gobierno que inspeccionaba la estructura de las agencias con responsabilidades en áreas operativas. Ello daba lugar a cambios estructurales significativos. Posteriormente se ha anunciado una reforma en profundidad en la Administración Pública con el objetivo de definir el papel que debe desempeñar el Estado.

\section{b) Gestión por resultados}

El proyecto más ambicioso es la introducción de un nuevo sistema de elaboración y gestión de presupuestos y en el marco de esta reforma la implantación de una gestión por resultados en las relaciones del Gobierno con las agencias. Se pide a las agencias que informen de los resultados que han conseguido a partir del presupuesto asignado.

\section{c) Limitación de costes}

Las agencias centralizadas desde el punto de vista financiero están limitadas en sus costes administrativos, de modo que si los costes salariales crecen por encima de lo previsto los responsables de la agencia tendrán que buscar la financiación adicional.

d) Política de personal

GuSTAFSSON señala que el número de empleados estatales ha disminuido en 112.000 en los últimos 10 años. De ellos, 40.000 fueron despedidos y 72.000 han pasado a agencias o empresas. Asimismo, la política de los salarios ha sido flexibilizada en el sentido de que se decida por las Agencias atendiendo a la valía de las personas y al mercado local (Gustafsson, 1995).

\section{Procesos de reforma en otros países}

Como en la mayoría de los países de la OCDE, en Francia, se está produciendo desde la segunda mitad de los años 80 una ralentización del montante de los recursos humanos. De hecho, entre 1985 y 1993, se ha estancado el número de funcionarios mediante la amortización de puestos. Asimismo, se ha iniciado una política de redistribución de personal, geográfica y funcional. A su vez, se han desarrollado los proyectos de servicio que analizan el funcionamiento de unidades administrativas y proponen objetivos a alcanzar.

Los gobiernos italianos han emprendido varias reformas, alentados por los fenómenos generalizados de corrupción y por el deterioro de la imagen de los funcionarios, debido a ciertas peculiaridades como son las jornadas reducidas de trabajo, la excesiva politización o el número excesivo.

Las medidas más significativas son la potenciación de los directivos profesionales, separando con nitidez entre la dirección política y la profesional, la laboralización de la gran mayoría de los empleados públicos y la generalización de la negociación colectiva, (Martínez Bargueño, 1995).

\subsection{Los programas de "modernización» en España}

Desde 1983, los sucesivos Gobiernos socialistas intentaron mejorar la Administración Pública en diferentes aspectos, que van desde la potenciación de la eficacia a las reformas organizativas y desde la redefinición de la distribución territorial de las competencias a la adaptación a las directrices de la Unión Europea. A tal efecto, elaboraron a lo largo de estos años varios proyec- 
tos de cambio, el último de ellos consistente en la aplicación de 204 medidas de modernización (BAÑón, 1993 y Parada, 1994). A continuación se analizan los aspectos más relevantes de cada uno.

Los programas de modemización se han desarrollado (según BAÑón) en tres etapas. La primera como una respuesta global a las insuficiencias de partida y como un cambio de cultura. La segunda desarrolla acciones concretas en materia de personal, organización y funcionamiento administrativo. La tercera se refiere a modificaciones internas de la organización, coordinación de relaciones con las administraciones central y autonómica y la vinculación de los tres niveles de gobiemo con la Unión Europea. De ahí que el concepto de modernización incluye: flexibilización organizativa y agilización de las relaciones con los ciudadanos, la redefinición de la distribución territorial del poder y la adaptación a los acuerdos comunitarios.

De modo resumido, podría decirse que en la primera etapa 1982-86 se adoptaron medidas de moralización y reforma de la función pública (Ley de Incompatibilidades y de reforma de la Función Pública), y en la segunda etapa se pretende adoptar el espíritu de las reformas de paises europeos (competitividad, nuevas tecnologías y cambio de cultura administrativa con el concepto de ciudadano-cliente), (BAÑÓN, 1993).

Pero ¿cuál ha sido el balance del desarrollo real de estas medidas?, o dicho de otro modo, ¿cómo se ha desplegado, al margen de intenciones, la política de la Administración? El despliegue de las reformas se sintetiza en seis puntos.

\subsubsection{Programa de recursos bumanos}

El sistema de función pública de 1978 se caracteriza por su debilidad y esta debilidad es fruto de la falta de asentamiento del modelo profesional debido a la difícil historia política de nuestro país en los últimos 75 años. Se suele considerar al Estatuto Maura de 1918 como el punto de origen del sistema profesional en la función pública española. Efectivamente en ese Estatuto se consagra la inamovilidad, las categorías y se da carta de naturaleza a los Cuerpos especiales.

En la Administración local se crean durante la Dictadura de Primo de Rivera los Cuerpos de Interventores, Secretarios y Depositarios, lo que supone un avance en la misma dirección de profesionalización. La Il República se caracteriza por la reducción de plantillas. Es especialmente significativa la Ley de Restricciones de Chapaprieta de 1934. Las características más significativas del franquismo hasta 1963 son las depuraciones y el reclutamiento partidista de la primera época, junto con la proliferación de los organismos autónomos. La legislación de 1963-64 ordena de algún modo la función pública creando órganos unificados, pero al mismo tiempo suprime las categorías e introduce el sistema de puestos, (BaEna del AlCázar, 1985, C. V).

Se podría decir que el sistema de función pública en 1978, se caracterizaba por los rasgos siguientes:

- Debilidad profesional derivada de los vaivenes de la historia del país.

- Inexistencia de carrera administrativa.

- Discrecionalidad en los nombramientos.

- Gran número de contratados e interinos seleccionados de modo arbitrario.

- Selección profesional para un número reducido de Cuerpos, que constituyen la columna vertebral de la Administración.

Se trata de un panorama muy diferente del existente en otros países. La función pública del Reino Unido había quedado asentada entre 1870 y 1920 y en Francia desde 1946.

Pero junto a la debilidad del sistema de función pública de la Administración Central, aparece la nueva organización territorial del Estado con unas Comunidades Autónomas que reciben un escaso personal procedente de la Administración Central. Por último, la Administración Local disponía de los Cuerpos nacionales y sólo en grandes municipios de personal técnico.

En esta situación los gobiernos democráticos de las tres Administraciones tienen un gran campo de actuación para actuar con gran libertad. Podríamos decir que salvo en determinadas zonas de la Administración Central, en el resto se produce en gran medida la ocupación por los políticos del entramado administrativo. Esta situación es muy característica de los años 80 en las tres Administraciones. En algunos casos, sigue en los primeros años 90 .

La Ley de medidas de reforma de la función pública de 1984 se asienta sobre tres principios: selección de personal en régimen laboral, sistema de promoción basado en el puesto de trabajo y movilidad generalizada del personal.

En cuanto al primer punto, habría que destacar el precepto de la ley 30/84, que atribuye al Ministerio para las Administraciones Públicas la tarea de especificar en las relaciones de puestos de trabajo aquellos puestos que, en atención a su naturaleza, se reservan a funcionarios públicos: Con ello se pretendía sustituir el régimen de funcionarios por el de laborales ${ }^{1}$. Aunque este proyecto fue declarado inconstitucional por el Tribunal Constitucional, continuó la contratación de personal laboral en funciones típicas de funcionario, sin respetar en muchos casos los principios de mérito y capacidad.

A su vez, se organizó la función pública en puestos de trabajo. De este modo, el sistema de puestos aparece como el pivote sobre el que se asienta la promoción de funcionarios y el 
salario. Este nuevo concepto, unido a la movilidad generalizada por interés del funcionario y a la libre designación para determinados puestos, da lugar a un sistema arbitrario y en constante movimiento, con el consiguiente resultado de un incremento de costos. Curiosamente para los puestos de nivel 28 e inferiores se establece una doble llave de inamovilidad, en el grado y en el puesto. De este modo se esclerotiza el sistema profesional haciendo inviable la movilidad por razones de la organización.

La consecuencia más importante de esta política la constituyó una fuerte desprofesionalización de la función pública, que se concretó en un cierto clientelismo. A ello respondió la libre designación de más de 2.000 puestos y los casi 800 puestos de eventuales. Como señala JimÉnEZ ASENSIO: -La debilidad de las estructuras burocráticas, la carencia de una función directiva profesionalizada ... resultaban el marco idóneo para el aterrizaje en bloque de la clase política sobre las estructuras administrativas. La función pública, una paupérrima función pública, apenas podría servir de dique de contención al empuje del poder político• (JIMÉNEZ ASENSIO, 1996, pág. 249).

En este sentido, es oportuno recordar que en la discusión del art. 103.3 de la Constitución, la frase eel acceso a la función pública de acuerdo con los principios de mérito y capacidad., sólo consiguió incluirse en el texto constitucional, tras un enconado debate en la Comisión. Finalmente se aprobó con la abstención del PSOE. (GaRRIDO FaLla, 1980).

\subsubsection{Política expansionista del sector público}

Durante los últimos veinte años se produjo un crecimiento espectacular del ratio Casto Público/PIB. Entre 1975 y 1995 este ratio pasó del $25 \%$ a un valor próximo al $50 \%$. Ciertamente otros países de la OCDE registraron un incremento del tamaño del sector público pero ninguno lo ha hecho con tal intensidad. "Una consecuencia derivada de esto es la dificultad para controlar y gestionar adecuadamente el gasto, porque el ritmo de crecimiento y la carencia de un cuadro de prioridades claro dificulta el control del proceso. (MONASTERIO, 1995, pág. 210).

Las manifestaciones de este fenómeno son patentes en el crecimiento de las prestaciones de la Seguridad Social, el apoyo a los sectores productivos, el aumento de los gastos de consumo y del empleo público y, en menor medida, el crecimiento de la inversión pública, escandalosamente reclamado por el desarrollo de la producción privada y al fin atendido desde 1989, cuando más comprometida era la situación presupuestaria. (Fuentes QuiNTANa, 1995, pág. 112).

\subsubsection{Indisciplina presupuestaria}

Señala FuENTES QUINTANa que sla voluntad política, proclamada en cada ejercicio presupuestario, de reducir el gasto público no constituye sino el abuso reiterado de una retórica estéril, y que la única solución del permanente problema del déficit público español y el aumento de su deuda resultará imposible si las instituciones y procedimientos presupuestarios que lo impulsan no se reforman. (Fuentes Quintana, 1995, pág. 147).

En nuestro país hemos pasado de una etapa de planificación integral y rígida a otra que podríamos calificar como de ausencia de planificación e incluso de previsión plurianual. Existía actividad planificadora, a veces importante, en diversos sectores -que ejercían los departamentos- pero ésta se desarrollaba en ausencia de un marco coordinado y armónico que aunase tanto las necesidades de los distintos sectores, como las de las Administraciones central y autonómica.

Ello hacía que llegasen al Consejo de Ministros, o incluso al Parlamento, ambiciosos planes elaborados por los responsables de cada sector, que no habían sido analizados con el rigor requerido por un órgano coordinador de la planificación, ni puestos en competencia con los planes o necesidades de otros sectores, por lo que no se disponía de un marco plurianual de necesidades reales ni de un orden de prioridades.

Por lo que respecta a la articulación de las actuaciones de la Administración Central y de las CC.AA., a pesar de algunos esfuerzos significativos, sobre todo por parte de los departamentos, los logros obtenidos fueron muy cortos. Uno de los aspectos en que esta articulación era más vital, era en materia de inversiones. La Unión Europea exige que los Planes que el Estado español presenta para ser objeto de cofinanciación comunitaria sean aprobados por los órganos responsables de la planificación y así lo han sido, según se desprende de la lectura de los mismos. Sin embargo, esta aprobación no supuso en aquellos años una revisión de su contenido.

La falta de coordinación de los planes sectoriales conducía a que muchos de éstos, aprobados por el Consejo de Ministros o por el propio Parlamento (LOGSE), sufrieran continuos aplazamientos en su calendario de ejecución o se quedasen en simples declaraciones de intenciones porque debían adaptarse a las dotaciones presupuestarias de cada año.

Por la misma razón, a lo largo del año eran aprobados paquetes de medidas no contemplados en los presupuestos, que generaban importantes modificaciones presupuestarias. Esta falta de horizonte plurianual y, en suma, de planificación, hacía que todo quedase condicionado a las dotaciones presupuestarias de cada año; es decir, eran papel mojado.

A falta de un plan estratégico y de programaciones plurianuales, el Presupuesto de cada año constituía el único marco 
de referencia del gasto público. Pero, además, en nuestro caso, éste arrastraba una serie de fallos que, de algún modo, limitaban su función:

- El sistema de asignación era incrementalista. Sobre la base del presupuesto del año anterior se realizaban las oportunas actualizaciones o se dotaban nuevas actuaciones, pero la base no es, generalmente, puesta en entredicho.

Esta práctica incrementalista se evidenciaba, igualmente, en los procedimientos de discusión del Presupuesto en el Parlamento. Ésta se realizaba por secciones presupuestarias (departamentos), siguiendo criterios exclusivamente orgánicos y económicos, pero no funcionales, como sería deseable.

Por la misma razón, uno de los documentos más valorados durante la discusión presupuestaria era el denominado "documento de diferencias", en el que se explicaban, partida a partida, las diferencias de dotación entre un año y otro. Habitualmente, los parlamentarios se interesaban más por las causas de las disminuciones que por los incrementos, consolidando así el tratamiento incrementalista del presupuesto.

- El proceso de elaboración del Presupuesto era muy corto. En los últimos años la Orden Ministerial con las instrucciones no solía publicarse hasta abril o mayo, por lo que las Oficinas Presupuestarias y el Ministerio de Economía y Hacienda disponían apenas de dos meses hábiles para hacer el presupuesto, y ello contando el mes de septiembre. Está claro que en este tiempo no era posible realizar ninguna labor de evaluación de la base, por lo que las posibles inconsistencias se arrastraban de un año para otro.

- La disciplina presupuestaria era muy laxa. La desviación media entre los créditos aprobados en el Parlamento y los definitivos sobrepasó el $24 \%$ durante los últimos años. (1989-1993).

En este sentido, José Manuel González Páramo, en un artículo dedicado al Presupuesto del año 1995 dice: *... es un hecho bien conocido que España tiene reglas y procedimientos presupuestarios que se encuentran entre los más laxos de la Unión Europea, junto con Bélgica y Grecia, (GONZÁl.EZ Páramo, 1995).

\subsubsection{Las ofertas de empleo priblico}

En el período 1985-1993 se aprobaron 228.978 plazas de oferta de empleo en la Administración del Estado (sin tener en cuenta Sanidad, Seguridad y Fuerzas Armadas). (Cuadro 5).
La media de oferta para el período 1985-1992 fue de 26.880, lo que supone un $5,6 \%$ del total de efectivos del colectivo, mientras que la tasa media de jubilaciones está en torno al 1,6\% (Cuadro 6). El exceso de oferta se comprueba para el mismo período, por ejemplo, en el caso de los maestros del área de gestión del MEC. Se ofertaron en ese período 15.298 plazas de maestros, dando lugar a un aumento neto de 8.703 personas, mientras que la evolución del número de alumnos de Enseñanza Primaria y Preescolar disminuyó en 256.889 en dicha etapa ${ }^{2}$.

\subsubsection{Planes de Empleo}

Para racionalizar los efectivos de personal se regularon los llamados "planes de empleo", que se concebían como un conjunto de medidas destinadas a determinar las necesidades de una organización en recursos humanos, teniendo en cuenta las funciones de su organización en un período de tiempo determinado. (Ley 22/1993, de 26 de diciembre).

El Reglamento General de Ingreso del personal al servicio de la Administración General del Estado establece una diferencia entre los planes de empleo integrales y los planes de empleo operativos. Los primeros especifican los objetivos a conseguir, los recursos humanos necesarios y las medidas de implementación, especialmente en materia de movilidad, formación y promoción. Los planes operativos tienen por objeto una mejor utilización de los recursos humanos y para ello establecen previsiones y medidas sobre movilidad, redistribución de efectivos y asignación de puestos.

Se trata, por consiguiente, de un instrumento que tiene como objetivo la optimización de los recursos humanos a través de procesos de redistribución. En este sentido, los Planes de Empleo plantean una reflexión estratégica sobre cada Ministerio, organismo o área administrativa con la finalidad de definir sus necesidades de personal. Esta reflexión puede conducir a una serie de medidas:

- Modificación de estructuras y de puestos de trabajo.

- Suspensión de incorporaciones de personal.

- Redistribución y reasignación de efectivos.

- Medidas de promoción interna.

- Prestación de servicios a tiempo parcial.

- Jubilaciones voluntarias y excedencias voluntarias incentivadas.

- Cursos de formación.

A. Palomar, sintetiza las medidas de planes de empleo en cuatro bloques: relativas a la movilidad, de dimensionamiento, de promoción interna y relativas a la jornada de trabajo. 


\section{(Cuarebro) 5}

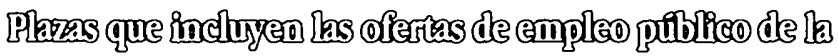

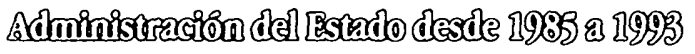

\begin{tabular}{|c|c|c|c|c|c|c|c|c|c|}
\hline & & Grapo A & Grapo B & Grapo C & Grupo D & Grupo E & $\begin{array}{c}\text { Total } \\
\text { funcionarios }\end{array}$ & $\begin{array}{l}\text { Personal } \\
\text { Eboral }\end{array}$ & $\begin{array}{l}\text { Total } \\
\text { gerteral }\end{array}$ \\
\hline (1) & 1985 & 4.979 & 10.537 & 6.293 & 6.294 & 800 & 28.903 & 10.388 & 39.291 \\
\hline \multirow[t]{6}{*}{ (2) } & 1986 & 4.635 & 9.461 & 3.404 & 4.300 & - & 21.800 & 9.963 & 31.763 \\
\hline & 1987 & 2.804 & 4.746 & 1.618 & 5.081 & - & 14.249 & 7.703 & 21.952 \\
\hline & 1988 & 3.599 & 5.196 & 2.397 & 3.318 & - & 14.510 & 5.997 & 20.507 \\
\hline & 1989 & 4.909 & 5.996 & 3.463 & 6.568 & - & 20.936 & 4.785 & 25.721 \\
\hline & 1990 & 4.969 & 5.450 & 2.460 & 5.015 & - & 17.894 & 8.732 & 26.626 \\
\hline & 1991 & 6.213 & 6.931 & 2.930 & 4.366 & - & 20.440 & 6.729 & 27.169 \\
\hline \multirow[t]{3}{*}{ (3) } & 1992 & 6.973 & 5.280 & 3.080 & 2.549 & 1.000 & 18.882 & 3.127 & 22.009 \\
\hline & 1993 & 4.345 & 2.998 & 1.965 & 2.657 & 1.000 & 12.965 & 975 & 13.940 \\
\hline & Total & 43.426 & 56.595 & 27.610 & 40.148 & 2.800 & 170.579 & 58.399 & 228.978 \\
\hline
\end{tabular}

(1) La Oferta de Empleo Público de 1985 incluia, además, 1.000 plazas para Universidades.

(2) La Oferta de Empleo Público de 1986 incluia, además, 2.071 plazas de Administración Local.

(3) El Acuerdo del Consejo de Ministros de 21 de junio de 1992 supuso la congelación de la convocatoria de 4.200 plazas sobre las inicialmente aprobadas para 1992.

\section{Ousactero (6)}

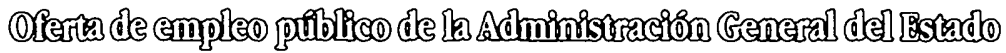

1983 \& 19292

\section{Comparación entre los efectivos existentes a diciembre de 1992 y la oferta media de empleo público de los años 1985 a 1992}

O.E.P. Media 1985-1992

Sulbsector

Administración del Estado y OO.AA.:

- Funcionarios

- Otros

Administración de la Seguridad Social

- Funcionarios

- Laborales

- Otros

- Funcionarios

- Laborales

- Otros

Totales parciales

- Funcionarios

- Laborales

- Otros

Total general
- Laborales

Cuerpos Docentes no Universitarios

\section{Efectivos}

NOTA: Los datos de efectivos están referidos al boletin estadistico del Registro Central de Personal de diciembre de 1992. No incluyen 125.888 efectivos de personal de Instituciones Sanitarias; 118.745 de F. y C. de Scguridad y 74.690 de F. Amadas.
1992

172.500

127.038

6.030

32.398

10.621

517

119.933

13.643

O.E.P. Total 1985-1992

92.258

48.121

11.532

22,4

24.763

3.095

11,5

9.303

1.163

40.593

5.074

18,9

324.831

137.659

20.190

482.680

157.614

19.702

73,3

57.424

7.178

26,7

26.880

100,0 
En definitiva, este doble proceso *permitiría escindir la reflexión en dos etapas formalmente diferenciadas: primera, que tendría por objeto determinar la existencia de necesidades, proceso al que pueden contribuir de forma muy eficaz los representantes de los empleados públicos cuyo ámbito de representación se corresponda estrictamente con el analizado; y, la segunda, en la que, una vez validado el diagnóstico, se efectúa una negociación sobre el conjunto de medidas con las que va a solucionarse el problema planteado, fase para la cual la representación centralizada adquiere una mayor consistencia, en razón a la necesaria homogeneidad que deben tener en su aplicación y a la necesidad de presentar este proceso como un desarrollo conjunto en el que una larga serie de medidas aparecen encadenadas" (PALOMAR, 1995).

\section{El desarrollo de los planes de empleo}

En febrero de 1996, se habían aprobado tres planes de empleo y estaban pendientes de aprobación otros tres. El examen de los mismos nos permite valorar en qué medida pudieron ser instrumentos de racionalización de personal. En el Cuadro 7 se detallan las características de los seis aprobados o presentados en febrero de 1996. Como resumen, se puede senalar lo siguiente:

1) Sólo el Ministerio de Defensa planteó la redistribución de personal sin un coste significativo.

2) INEM y Correos no hacían análisis alguno de las necesidades de personal y se limitaban a señalar que se mantiene la cifra de personal.

3) En todos los planes, salvo en Defensa, se planteaban aspectos de fijeza de personal, de funcionarización, de promoción interna y de oferta de empleo.

4) El MOPTMA proponía la redistribución de 2.692 efectivos y la jubilación de 1.117. Como contrapartida planteaba modificaciones que suponen un coste de 4.555 millones de ptas. En concreto, proponía un incremento salarial del 9,28\% para el personal laboral. (МотмA, 1995).

5) En conjunto, los seis planes de empleo citados suponían un coste superior a 8.000 millones de ptas.

Se podría decir que los planes de empleo, nacidos para lograr una redistribución de personal en un contexto de mayor eficacia de la Administración Pública, se convirtieron en un mecanismo de mejora salarial por diferentes vías, sin un análisis suficiente de dimensionamiento.

En este sentido, es bueno recordar que el entonces Director General de la Función Pública señalaba que alos planes de empleo habrán de tener una incidencia positiva sobre los Presu- puestos Generales del Estado en una triple dirección: minoración de la presión sobre el gasto público derivada del control del crecimiento inercial de recursos humanos, racionalización de las decisiones presupuestarias sobre los créditos de personal y aumento de la capacidad de maniobra del Presupuesto en la asignación de recursos en el cap. I. (González GallarDo, 1994, pág. 259).

Como resumen, se puede decir que, aunque técnicamente la elaboración de planes de empleo podría ser válida, su aplicación resultó un fracaso. Apenas incidieron en el análisis de dimensionamiento de personal y consiguiente redistribución y, por el contrario, estimularon deslizamientos salariales a través de medidas marginales.

\subsubsection{Medidas de descentralización}

- La Administración propició en esta etapa la creación de entes públicos de estatuto especial. Estos entes se caracterizaban porque prestaban servicios en régimen de monopolio, con una regulación estatutaria atomizada que les exceptuaban de algunas normas de gestión, con la finalidad, como señalan algunos autores, de sacarlos fuera del imperio del Derecho. Los resultados de esta nueva forma de gestión han sido la selección poco rigurosa de personal, sobre todo, en niveles altos y una gestión con normas de Derecho privado. Todo ello se ha traducido, en ocasiones, en una toma de decisiones muy peculiar (MEDELA, 1993 y SAZ, 1994).

En definitiva, estamos ante una peculiar concepción de la modemización que si, por un lado, se plantea dar respuesta a problemas reales como la necesidad de abordar cambios derivados de la dificultades del Estado de Bienestar o de la adaptación de la Administración al Estado de las Autonomías o a la Unión Europea, establece, por otro, algunos mecanismos organizativos o de gestión que ponen en peligro la transparencia de la actividad pública. Como señala S. del SAZ, se ha pasado en la actualidad a una aplicación indiscriminada del Derecho privado que se concreta en la huida de los procedimientos administrativos de control del gasto, en la disminución de los controles y garantías para la enajenación de bienes patrimoniales y en la fuga de los procedimientos de selección de contratistas y régimen de funcionarios. (SAZ, 1994) ${ }^{3}$.

Outo de los aspectos típicos de la emodernización. es el cambio de cultura administrativa. En este sentido, se acentúa la idea del ciudadano como cliente. o la concepción de la responsabilidad por los resultados. A su vez, se pone el énfasis en técnicas de gestión para aumentar la eficacia. En este contexto se desarrollan cursos de planificación estratégica, de dirección por objetivos, de técnicas de negociación, de auditorías y otros similares. 


\begin{tabular}{|c|c|c|c|}
\hline \multicolumn{4}{|c|}{$\begin{array}{l}\text { Cuadro } 7 \\
\text { slanes de Empleo presentados }\end{array}$} \\
\hline CONCEPTO/PLAN EAPLEO & DEFENSA & NEM & CORREOS \\
\hline $\begin{array}{l}\text { Denominación especifica del } \\
\text { Plan de Empleo }\end{array}$ & P. de E. operativo del MD & P. de E. del LNEM. & $\begin{array}{l}\text { P. de E. del Org. Auro. Correos y } \\
\text { Tekégrafos. }\end{array}$ \\
\hline Fase de tramitación & $\begin{array}{l}\text { Aprobado, Res. de } 26-12 \cdot 1995 . \\
\text { Publicado, BOE } 28-12-1995 \text {. }\end{array}$ & $\begin{array}{l}\text { Aprobado. Res. de 196-1995. } \\
\text { Publicado, BOE 23-6-1995. }\end{array}$ & $\begin{array}{l}\text { Aprobado, Res. 26-12-1995. } \\
\text { Publicado, BOE 28-12-1995. }\end{array}$ \\
\hline Periodo de vigencia & 1 año descle su publicación. & 2 años desde su publicación. & 3 años descle su aprobación. \\
\hline Ámbito de aplicación & $\begin{array}{l}\text { - Personal crill funcionario y laboral. } \\
\text { - Miniserio y sus 00..A. }\end{array}$ & Planulla del LVEM. & $\begin{array}{l}\text { Plancilla de 65.500 efectivos (fijos y } \\
\text { temporales). }\end{array}$ \\
\hline Compromiso/Objetivos & Elaboración de un $P$. de E. integral & $\begin{array}{l}\text { No phantear aumentos de a plantilla: } \\
15.204 \text { efectivos a 22-2-1995. }\end{array}$ & $\begin{array}{l}\text { Planificación intema de los RR.HH. a través } \\
\text { de Planes operativos de Empleo. }\end{array}$ \\
\hline $\begin{array}{l}\text { Conversión de Empleo temporal } \\
\text { en fijo }\end{array}$ & No & $\begin{array}{l}\text { - de funcionarios interinos. } \\
\text { - de contratados por obra o servicio, que } \\
\text { realiza funciones permanentes. } \\
\text { El } 16-11.95 \text { : convocat. de } 1.900 \mathrm{Ax} \text {. Labor. }\end{array}$ & $\begin{array}{l}\text { Sin aumentar efectivos, } 1^{\top} \text { ano, concurso- } \\
\text { oposición de: } 1.750 \text { plazas Aux. Postales, } \\
250 \text { Ajud. y } 500 \text { pers. rural y vario. }\end{array}$ \\
\hline Funcionarización & No & $\begin{array}{l}\text { Del personal laboral fijo, a dos grupos de } \\
\text { tifulación. Convocatorias } 1995 \text { y } 1996 \text {. }\end{array}$ & $\begin{array}{l}\text { A grupo D categorias laborales de Aux. y } \\
\text { Oficial Admvo. } \\
\text { De taboral fijo con fun. propias de } \\
\text { funcionario. }\end{array}$ \\
\hline Promociōn interna & No, diferida a un P. de E. integral. & $\begin{array}{l}\text { Plazas: } 150 \text { al A, } 75 \text { al B y } 200 \text { al C. } \\
\text { Coste } 132 \text { Mill. ptas. } 1996 . \\
\text { Convocatorias } 50 \% 1995 \text { y } 50 \% 1990 .\end{array}$ & $\begin{array}{l}\text { Convocatorias especificas } \\
1995,2.000 \text { plazas C. Ejecuuvo } \\
1996,3.000 \text { plazas C. Ejecutivo } \\
\text { Promoción de pers. laboral fijo }\end{array}$ \\
\hline Oferta de Empleo Público & No & No & No \\
\hline Reasignación de efectivos & En las 3 fases del ant. 20.1,g) Ley 30/1984. & $\begin{array}{l}\text { - Por redinensionamiento de la red de } \\
\text { oficinas. } \\
\text { - No se dispone regulación especifica. }\end{array}$ & $\begin{array}{l}\text { - Por desequilibrios internos, nuevo plan } \\
\text { de transpones y nuevas tecnologias. } \\
\text { - No se regub el procedimiento, remite al } \\
\text { Reglamento del Personal al servicio del O. Auth } \\
\text { Cy T-RD 1638/1985, de } 6 \text { de octubre. }\end{array}$ \\
\hline Excedencias & $\mathrm{Si}_{\text {, forzosa } y}$ voluntaria incentivada remite a la ley $30 / 1984$. & No & No \\
\hline Jubilación anticipada incentivada & Si, an 34 Ley 30/1984. & No & No \\
\hline $\begin{array}{l}\text { Traslados } \\
\text { - Cambio de residencia } \\
\text { - Movilidad }\end{array}$ & $\begin{array}{l}\text { - Por reasignación con cambio de residencia y por } \\
\text { movilidad por cambio de adscripción del puesio. } \\
\text { - Indemnizaciones previstas en el P. de E. y además las } \\
\text { del RD 236/1988. }\end{array}$ & No se dispone regulación especifica. & $\begin{array}{l}\text { No se regula el procedimento, remite al } \\
\text { Reglamento del Personal al Servicio del O. } \\
\text { Aut. C. y T-RD } 1638 / 1985 \text {, de } 6 \text { de octubre. }\end{array}$ \\
\hline Coste del Plan & No tiene & $\begin{array}{l}\text { Pro interna } 132 \text { Mill. } \\
\text { El resto no se determina. }\end{array}$ & $\begin{array}{l}\text { Promo. D a C } 1.200 \text { Mill, A. Telecom. } 175 \\
\text { Mill. El resto no se determina. }\end{array}$ \\
\hline
\end{tabular}




\section{Conclusiones}

El balance de trece años de reformas administrativas sugiere la dificultad de adoptar determinadas medidas de flexibilización de la gestión, sin hacer un análisis preciso del nivel de solidez de la Administración afectada. En este sentido los patrocinadores de la :modernización. han puesto el énfasis en medidas a veces arriesgadas desde el punto de vista de la transparencia.

Ello, sin embargo, no debería dar lugar a la defensa de las posiciones que defienden autores anclados en el modelo administrativo a ultranza, para quienes el uniformismo jurídico es un manto omnicomprensivo que resuelve todos los problemas. Como señala R. BAÑON, el miedo a los comportamientos corruptos, simultáneamente es como una fuerza de resistencia a las innovaciones que propugnan el aumento de la discrecionalidad de actuación, la flexibilidad organizativa• (BAÑón, 1993).

De ahí que las conclusiones de estas reformas transmitan una sensación agridulce de medidas positivas en el enfoque y en el cambio de cultura, junto a otras poco elaboradas que sugieren una huida hacia adelante, más que un plan coherente de cambio. Estas conclusiones se sintetizan en cinco puntos:

\subsection{Cambios en la cultura administrativa}

Las políticas de modernización a partir de 1986 incorporaron cambios en la cultura administrativa. Ello significó, por una parte, la publicación de libros y artículos y la realización de seminarios sobre las políticas de modernización en el área de la OCDE. Por otra parte, se multiplicaron los cursos de formación de funcionarios en técnicas privadas y se impulsaron las medidas de mejora de atención al ciudadano. Al mismo tiempo, se produce en esta época un gran impulso a las tecnologías de la información.

\subsection{Una reforma al margen de los funcionarios}

La selección de los empleados públicos antes de 1978, salvo algunos cuerpos del Grupo A, careció del rigor mínimo exigido en países de la Europa Occidental. De ahí la gran debilidad de la función pública española a finales de los 70. La Ley 30/84 de Reforma de la Función Pública y su desarrollo posterior no mejoró la situación heredada. Esta debilidad dio lugar a una ocupación de puestos de trabajo de nivel superior por procedimientos ajenos al principio del mérito y por consiguiente a una cierta politización de las Administraciones Públicas, Central, Autonómica y Local en los años 80.
Este hecho unido a la flexibilización de la gestión y a la creación de entes públicos exentos originó problemas en la gestión ordinaria. Se evidencia que el principio de la responsabilidad exige una función pública estrictamente profesional y una Administración en la que funcione el principio de frenos y contrapesos.

\subsection{Indisciplina en la politica del gasto público}

La desviación media entre los créditos aprobados en el Parlamento y los definitivos sobrepasó el $24 \%$ durante los últimos años (1989-1993). Este hecho hizo decir a G. PÁramo que *España tiene reglas y procedimientos presupuestarios que se encuentran entre los más laxos de la Unión Europea, junto con Bélgica y Grecia. (G. PÁramo, 1995).

En estos años predominó la cultura del gasto sobre la cultura de la eficiencia. En este sentido, la política de reformas de la maquinaria administrativa estuvo alejada de las pautas de la política económica.

\subsection{Creación indiscriminada de empleo público}

Otra característica de la época 1983-1996 es la creación de empleo público más como política para reducir el paro, que como política de cobertura de necesidades de personal. La media de oferta de empleo público de la Administración Central en el período $1985-1993$ fue de 26.880 , un 5,6\% del total de efectivos del colectivo, mientras que la media de las jubilaciones estaba en torno al 1,6\%. Si a estos datos añadimos las ofertas de Sanidad, Seguridad y Fuerzas Amadas más personal contratado e interino, la suma total media anual se acerca a 40.000 personas. Esto se producía mientras la Administración Central estaba transfiriendo competencias a las Comunidades Autónomas. Los "planes de empleo" trataron de dar una solución a este problema mediante políticas de redistribución de efectivos. Desgraciadamente, su éxito ha sido muy escaso.

\section{5. .La huida del Derecho"}

En esta etapa proliferaron los organismos públicos exceptuados de normas fundamentales de Contratos del Estado, de los procedimientos de gestión del Presupuesto o de control del gasto. Cada Ente adoptaba una regulación específica :a medida", casi siempre sobre el principio de prescindir de enojosos trámites burocráticos. Ello dio lugar a que nuestros Entes tuvieran en ese momento una de las normativas más flexibles de la Unión Europea. Al mismo tiempo la flexibilización del sistema de control, sin el recambio de un adecuado mecanismo de au- 
ditoría periódica, agudizó la carencia de niveles mínimos de control del gasto.

Si a todo esto añadimos la falta de solidez de la Administración española heredada del franquismo y, en particular, la ine- xistencia de una función directiva formalizada, no es de extrañar que existieran problemas de transparencia y de eficiencia en el uso de los recursos.

\section{- Director General del Boletin Oficial del Estado.}

' A lo largo de la década pasada se han desanollado teonias que imataban de justificar lo que podriamos llamar la eprivatización. del servicio público, que se concretaban en la defensa de la contratación laboral, en la politización de la función direciva, en la movilidad indiscriminada del personal y en la crítica sistemática a la separación entre política y administración. Esta tendencia ha seguido en los últimos años y un buen ejemplo de ello es un arúculo de Francisco LovGo. A parir de un análisis de la situación actual que puede ser comparido en buena medida, éste propone cuatro puntos básicos para una reforma de la función pública. Estos criterios son los siguientes:

1) Supresión del sistema de Cuerpos y acceso directo a través de puestos de trabajo.

2) Establecimiento de aciertas modulaciones específicas de los supuestos de suspensión de la relación de empleo contemplados por la norma laboral: que sería aplicable al empleo público.

3) Regulación especifica para el empleo público de la concurrencia de las causas económicas, técricas, objetivas o de producción del Estatuto de los Trabajadores.

4) El nuevo estatuto del empleo público debe facilitar el ejercicio de la función directiva, si bien la referencia a una carrera directiva dentro de la función pública podría abonar interpretaciones de signo endogámico que personalmente no compartiría. (LONGO, 1995)

- Aunque las autoridades del MAP afirmaron que el volumen global de oferas de empleo era inferior a las necesidades de reposición, los datos disponibles no avalan esta afimación. La oferta de empleo aprobada para 1996 constaba de 4.422 puestos para un colectivo de 470.968 personas (excluidos Justicia, FF.AA., Seguridad del Estado, Instituciones Penitenciarias y Universidades). Representaba, por tanto, un porcentaje del $0,98 \%$ respecto del colectivo. Si a la cifra de ofera, añadimos la ofera oculta a través de autorizaciones de los Ministerios de Hacienda y de Administraciones Públicas, la cifra se eleva a 4.679. (Quedarian todavía los incrementos que producen los Entes Públicos). Por tanto, el porcentaje se eleva, al menos, a un 0,99\%.
Ahora bien, las jubilaciones de este colectivo, para 1996, obtenidas a través de una muestra de 216.072 personas, arrojan un porcentaje de $0,95 \%$. Por consiguiente la oferta de empleo supera ligeramente la cifra de personal de reposición. En anos anteriores la diferencia es muy importante a favor de las ofertas de empleo. Por lo demás, este dato se confirma si observamos la serie estadistica de personal publicada por el Registro del MAP:

$\begin{array}{cccccc}\mathbf{1 - 1 . 9 0} & \mathbf{1 - 1 - 9 1} & \mathbf{1 - 1 . 9 2} & \mathbf{1 - 1}-93 & \mathbf{1 - 1}-94 & \mathbf{1 - 1}-95 \\ 2.157 .341 & 2.170 .937 & 2.193 .660 & 2.244 .908 & 2.264 .741 & 2.298 .288 \\ & 0,6 \% & 1,0 \% & 2,3 \% & 0,9 \% & 1,5 \%\end{array}$

(La cifra oficial del Registro de 1-1-95 es de 1.992 .616 porque en esa fecha decidió prescindir del personal de empresas públicas. Realizada la homologación con los años anteriores resulta una cifra de 2.298 .288 efectivos en ese año).

' Señala en este sentido S. SAZ que -sería necesario, en definitiva, según esta opinión compartida, revisar la categoría de las entidades de Derecho público, encuadrándolas entre los Organismos Autónomos sin perjuicio de que una parte de su actividad delimitada por la Ley, quede sometida al Derecho privado, con lo que se salvaguardarian para el Derecho público los procedimientos de preparación de los contratos y selección de contratistas. Asimismo, habría que excluir de este tipo aquellos supuestos cuyo único cometido consiste en ejercer funciones públicas o de autoridad.

Al otro lado, el limite a la creación de sociedades mercantiles en que parecen pugnar por convertirse los grandes servicios públicos prestados por la Administración en régimen de gestión directa (correos, puertos, aeropuertos, ferrocarriles, etc.) debe situarse, según SALA ARQUER, en la posibilidad de que exista una actuación sometida a una real y efectiva competencia, ya que el recurso a la forma societaria o al somecimiento de la mayor parte de su acividad al Derecho privado no está justificado cuando las pretendidas empresas desarmollen su acividad en un régimen de monopolio o cuasi monopolio. (SAz, Silvia, 1994, pág. 82).

\section{Bibliografía}

ARENiLA, M. (1995): -Los Recursos Humanos en las Administraciones Püblicas, en La estructura de la Administración Prublica: análisis, evaluación y propuestas. Madrid, Tecnos.

Baena del AiḉzAR, M. (1985): Curso de ciencia de la Administración, Madrid, Tecnos. BANON, R. (1993): -La modernización de la Administración Pública. Balance y perspectivas. Revista Politica y Sociedad, 13, Madrid.

BAFris, J. (1995): •El sector público espanol ante la integración europea., en Problemas económicas españoles en la década de los 90 . Galaxia e Informe sobre Ejecución del Presupuesto del Estado. Ministerio de Economia y Hacienda, 1993.

Butrer, R. (1993): -The evolution of the Civil Service. A Progress Report. Public Administration, Vol. $71, n^{n} 3$.

CRUz FERRER, Juan (1984): La funciōn puiblica superior en Estados Unidos. INAP. Madrid.

Fuenitis Quintana, E. (1995): Problemas económicas cspañoles en la década de los 90 . Ed. Galaxia Gutemberg. Madrid.
Garrido Falla, F. (1980): Comentarios a la Constitución. Ed. Civitas. Madrid.

GONZÁlEZ Gallardo, L. (1994): •Introducción de sistemas de planificación de recursos humanos y flexibilización de la relación de empleo en el ámbito de las Administraciones Públicas. Revista Presupuesto y Gasto Priblico, n. 12.

Gonzáez Páramo, José Manuel (1995): •Presupuestos Generales del Estado para 1995: El dificil anclaje de la credibilidad presupuestaria., Cuaderno de Información Económica, n. 91.

GUSTAFSSON, G. (1995): :Participación y racionalización en el área de recursos humanos: reforma de la gestión y el empleo público en Suecia. Gestión y Análisis de Politicas Priblicas, n. 2 .

HooD, Cristopher (1994): •l'évolution de la gestion publique au Royaume-Uni et la suppresion des privilèges de la fontion publique, Revrue Francaise d'Administration Publique.

INAP (1992): Plan de modernización de la Administración del Estado. Madrid.

INE: Anuario Estadistico, 1994. 
INSTTTUTO DE ESTUDIOS FISCALES (1993): Estructura institucional y gestion del gasto puiblico de algunos paises de la OCDE Madrid.

JimÉnEZ ASENSIO, Rafael (1996): Altos Cargos y Directivos Priblicos. I. Vasco de Administración Pública. Onaati.

JONES, G.W. (1993): •la modemización administrativa en el Reino Unido: una perspectiva general. Revista Politica y Sociedad, n." 13, 1993, Madrid.

LoNGO, F. (1995): •Reforma del empleo público: Tótem y Tabú., en Gestión y análisis de Politicas Priblicas, $n{ }^{\circ} 2$.

Martínez BarGueño, M. (1995): .la reforma de la relación de empleo público en italia. Gestión y Análisis de Politicas Püblicas, n. 2.

MEDEL, Pilar (1993): -Los entes públicos en España. Una propuesta de reforma. Revista del Presupuesto del Gasto Priblico, n." 11.

MéNY, Y.W WIGHT, V. (1994): La riforma anministrativa in Europa, Bolonia, Il Mulino.

METCALfE, les y RICHARDS, Sue (1993): La Modemización de la Gestión Priblica. Instituto Nacional de Administración Pública. Madrid.

MONASTERIO ESCUDERO, E. (1995): -Una política presupuestaria para alcanzar el déficit de convergencia en 1999. Revista Presupuesto y Gasto Público, n. 15.

MOPTMA: Plan de Empleo, 27 de diciembre de 1995. (Primer proyecto).
OCDE (1996): España, 1996, Mundi Prensa, Madrid.

Palomar, A. (1995): :Planes de Empleo y Reforma Administrauva. Gestion y Análisis de Politicas Públicas, n. ${ }^{\circ} 2$.

Parada VúzQuez, R. (1994): Onganización y Empleo Puiblico. M. Pons. Madrid.

PETERS, Guy (1994): L'échec du managerialisme dans une société manegeriale: la gestión du secteur public aux Etats-Uniso. Rerue Francaise d'Administration Publique.

Programa de Convergencia 1995-97.

Revista Internacional de Ciencias Adminstrativas, n. ${ }^{\circ} 3$ (1991). Análisis de la Función Pública en Alemania, Australia, Canadá, Francia, Gran Bretaña y Estados Unidos.

SAz, Silvia (1994): ‘la huida del Derecho Administrativo: últimas manifestaciones. Aplausos y críticas. $R A P, \mathrm{n}^{\circ} 133$.

SEAGE, Julio (1996): -Diez Medidas para un programa de reformas de la Administración Pública. Nueva Revista, n. 43.

L. SмmH, Bruce (1984): The Higher Civil Service in Europe and Canada. The Brookings Institution. Washington.

STEVENS, A. (1990): -Les Agences d'exécution d'administration et leur impact sur le $\mathrm{Ci}$ vil Service. Revue française d'administration publique, n. 55. 